\title{
Analytic Semigroup Generated by the Linearization of a Riemann-Dafermos Solution
}

\author{
Xiao-Biao Lin \\ Communicated by Charles Li, received March 15, 2004.
}

\begin{abstract}
Dafermos regularization is a viscous regularization of hyperbolic conservation laws that preserves solutions of the form $u=\hat{u}(X / T)$. A RiemannDafermos solution is a solution of the Dafermos regularization that is close to a Riemann solution of the conservation law. Using self-similar coordinate $x=X / T$, Riemann-Dafermos solutions become stationary. In a suitable Banach space, we show that the linear variational system around such solution is sectorial, thus generating an analytic semigroup.
\end{abstract}

\section{Contents}

1. Introduction

2. The Middle Region 198

3. The Side Regions 200

4. Matching of solutions in the middle and side regions 203

References

\section{Introduction}

A Riemann solution $u=\hat{u}(X / T)$ is a solution to the Riemann problem of the conservation law

$$
u_{T}+f(u)_{X}=0, \quad u(X, 0)=\left\{\begin{array}{l}
u^{\ell}, \text { if } X<0, \\
u^{r}, \text { if } X>0 .
\end{array}\right.
$$

Dafermos regularization $[\mathbf{4}, \mathbf{2 7}, \mathbf{2 8}, \mathbf{2 9}]$ is a viscous perturbation to the conservation law that preserves solutions of the form $u=\hat{u}(X / T)$ :

$$
u_{T}+f(u)_{X}=\epsilon T u_{X X} .
$$

1991 Mathematics Subject Classification. Primary 35; Secondary 46.

Key words and phrases. Dafermos regularization, hyperbolic conservation law, RiemannDafermos solution, analytic semigroup. 
Solutions of the Dafermos regularization that is near a Riemann solution of conservation law and assume the form $u=\hat{u}(X / T)$ will be called Riemann-Dafermos solutions. It is known that under some general conditions, any structurally-stable Riemann solution [23] consisting entirely of shock waves has a nearby RiemannDafermos solution [24]. Szmolyan proved that for small $\epsilon$, structurally-stable, classical Riemann solutions which consist of $n$ rarefactions and Lax shock waves have Riemann-Dafermos solutions nearby [26]. The proof of these results uses geometric singular perturbation theory [8].

Motivated by these results, in this paper, we consider Riemann solutions that may have shock and rarefaction waves in a bounded region $-\bar{x}<X / T<\bar{x}$, and constant values for $|x|>\bar{x}$. The nearby Riemann-Dafermos solutions may have internal layers in $|x|<\bar{x}$, and are near constants for $|x|>\bar{x}$.

In the similarity variable $x=X / T, t=\ln T$, the Riemann-Dafermos solution, denoted by $u(x, \epsilon)$ becomes a stationary solution to the Dafermos regularization in $(x, t)$ coordinates:

$$
u_{t}+f(u)_{x}-x u_{x}=\epsilon u_{x x} .
$$

We are interested in the stability of solutions of the Dafermos regularization with initial conditions close to the stationary solution $u(x, \epsilon)$. Spectrum of such solutions has been studied in $[\mathbf{2 1}]$. It has been shown that in suitable function spaces, the essential spectrum is bounded to the left of the line $\operatorname{Re} \lambda \leq \delta_{0}$ for some $\delta_{0}>0$. Thus, the spectrual stability is determined by eigenvalues. Using singular perturbation method, asymptotic expansions of eigenvalue/eigenfunctions have been obtained. Whether or not the spectrual stability implies linear stability was not addressed in that paper.

Note that for a single viscous shock of the usual regularization

$$
u_{T}+f(u)_{X}=u_{X X},
$$

Evans function and the related gap lemma $[\mathbf{7}, \mathbf{1 0}]$ have been major tools in studing the eigenvalues.

Let $t=\epsilon \xi, x=\epsilon \xi$.

$$
u_{\tau}+f(u)_{\xi}-\epsilon \xi u_{\xi}=u_{\xi \xi}
$$

The linear variational system around $u_{\epsilon}(\xi)=u(\epsilon \xi, \epsilon)$ is

$$
U_{\tau}=U_{\xi \xi}-D f\left(u_{\epsilon}\right) U_{\xi}-\left(D f\left(u_{\epsilon}\right)\right)_{\xi} U+\epsilon \xi U_{\xi},
$$

which can be written as

$$
U_{\tau}=\mathcal{A} U
$$

The spectrum problem $(\mathcal{A}-\lambda) U=h$ for $(1.4)$ is

$$
U_{\xi \xi}-D f\left(u_{\epsilon}\right) U_{\xi}-\left(D f\left(u_{\epsilon}\right)\right)_{\xi} U+\epsilon \xi U_{\xi}=\lambda U+h(\xi) .
$$

For any $0<\delta<\pi / 2$ and $a \in \mathbb{R}$, let

$$
\begin{array}{r}
\Sigma_{\delta}=\{\lambda:|\arg (\lambda)| \leq \pi / 2+\delta\}, \lambda \neq 0, \\
\Sigma_{\delta}+a=\{\lambda:|\arg (\lambda-a)| \leq \pi / 2+\delta\}, \lambda \neq a .
\end{array}
$$

We call a linear operator $A$ in a Banach space $X$ a sectorial operator if it is a closed, densely defined operator, and there exist some $M \geq 1$ and $a \in \mathbb{R}$ such that

(i) for some $0<\delta<\pi / 2$ and $a \in \mathbb{R}, \Sigma_{\delta}+a \subset \rho(A)$; 
(ii) there exists a constant $M$ such that

$$
\left\|(\lambda-A)^{-1}\right\| \leq \frac{M}{|\lambda-a|} \quad \text { for } \lambda \in \Sigma_{\delta}+a, \lambda \neq a .
$$

See $[\mathbf{6}, \mathbf{9}, \mathbf{2 0}]$ for reference of the sectorial operators and related analytic semigroups.

The main goal of this paper is to show that $\mathcal{A}$ is sectorial in a Banach space of continuous functions with weighted norm. Then classical results imply that $\mathcal{A}$ generates an analytic semigroup $T(t)$. The result obtained in this paper has many important consequences:

(1) The existence of solutions to the initial value problem (1.3) near $u_{\epsilon}$ in a suitable function space can be proved by a standard contraction mapping argument if $T(t)$ is an analytic semigroup $[\mathbf{9}]$. The function space for the initial data can be the fractional power of Banach spaces $\mathcal{A}^{\alpha}, 0<\alpha<1[\mathbf{9}, \mathbf{2 0}]$ for semi-linear parabolic equations, or the intermediate spaces introduced by Da Prato and Grisvard for fully nonlinear parabolic equations $[\mathbf{1}, \mathbf{5}, \mathbf{1 5}]$.

(2) If $\operatorname{Re} \sigma(\mathcal{A})<-a, a>0$, then $T(t) U_{0} \leq M e^{-a t} U_{0}$. Thus, linear stability of the Riemann Dafermos solution is determined by its spectrual stability.

A series of papers by Liu, Zumbrun, and Howard shows that linear stability implies nonlinear stability $[\mathbf{1 7}],[\mathbf{1 8}],[\mathbf{1 6}],[\mathbf{3 0}]$.

The stability of Riemann-Dafermos solutions is not only of independent interest, but also closely related to the stability of the Riemann solution of conservation laws and stability of the viscous shock solution of the usual viscous regularization (1.2). For a system of two equations, stability conditions of Riemann-Dafermos solutions near two Lax shocks has been obtained [21]. It has been shown that the stability condition is precisely the same for the stability of large shocks obtained by Schochet and Lewicka on hyperbolic conservations laws [12, 25]. Recently, for large noninteracting shocks, Lewicka showed that the BV and $L^{1}$ stability conditions for hyperbolic conservation laws are equivalent to $\operatorname{Re} \lambda<0$ and $\operatorname{Re} \lambda<1$, respectively, for the eigenvalues of corresponding Riemann-Dafermos solutions [13].

In the similarity coordinate $x=X / T$, Dafermos regularization can also be viewed as an asymptotic approximation to the usual regularization (1.2) for large $T$. Using the change of variables $x=X / T, t=\ln T$ to (1.2), we obtain

$$
u_{t}+(D f(u)-x I) u_{x}=e^{-t} u_{x x} .
$$

If $t$ is large, $e^{-t}$ is small and is slow varying. If we freeze $t=t_{0}$ and let $\epsilon=e^{-t_{0}}$, then we have (1.1), which is a good approximation in a time interval where $e^{-t}$ is close to $e^{-t_{0}}$. In the self-similar variable $x=X / T$, Riemann solutions appear to be asymptotic states of solutions of (1.6). If for small $\epsilon$ the Riemann-Dafermos solution is stable, then by piecing together such solutions on time intervals where they approximate solutions to (1.6), we have a pseudo orbit - the terminology used in the classical shadowing lemma. Following the idea that proves the shadowing lemma, we may be able to show that in the self-similar coordinate $x=X / T$, solutions of (1.6) will approach Riemann solutions as $t \rightarrow \infty$.

For the problem at hand, it suffices to show that $U_{\xi \xi}+\epsilon \xi U_{\xi}$ is sectorial. The other terms can be treated as perturbations to the sectorial operator, resulting another sectorial operator [20]. For completeness, we include the perturbation terms and show that they can be handled by a contraction mapping defined by 
integral equations. Besides, these detailed computation may offer clues for finding a better weight function in our future work.

Let $E$ be the Banach space of bounded continuous functions $U(\xi)$ on $R$ with the weighted norm

$$
\|U\|_{E}=\sup _{|\xi| \geq \bar{x} / \epsilon}\left\{|U(\xi)| e^{\epsilon \xi^{2} / 4}\right\}+\sup _{|\xi| \leq \bar{x} / \epsilon}\{|U(\xi)|\}<\infty .
$$

TheOREM 1.1. Let $f \in C^{3}(\mathbb{R})$, a bounded function with up to third bounded derivatives on $\mathbb{R}$. For the Riemann-Dafermos solution $u_{\epsilon}(\xi)=u(\epsilon \xi, \epsilon)$, assume that there exists $\bar{x}>0$ such that for $|\xi| \leq \bar{x} / \epsilon,\left|u_{\epsilon}\right|_{C^{2}} \leq C$ uniformly for all $0<\epsilon<\epsilon_{0}$ while for $|\xi| \geq \bar{x} / \epsilon,\left|u_{\epsilon}\right|_{C^{2}}=O(\epsilon)$. Consider the spectrum problem $(\mathcal{A}-\lambda) U=h$ as in (1.5) in the space $E$. Then $\mathcal{A}$ is a sectorial operator in $E$.

The main tools used in this paper are exponential dichotomies and shadowing lemmas $[\mathbf{3}, \mathbf{1 9}, \mathbf{2}, \mathbf{1 4}, \mathbf{2 2}]$ in the dynamical systems theory developed to study time stability problems. Recently, it has been found useful on boundary value problems with one spatial dimension. This paper is one more example.

The choice of the weight function $w(\xi)=e^{-\epsilon \xi^{2} / 4}$ used in the space $E$ is aimed to maximize the smallest eigenvalue in norm as $\xi \rightarrow \pm \infty$. Consider the eigenvalue $\mu$ for the second order system

$$
\begin{array}{r}
U_{\xi \xi}+\epsilon \xi U_{\xi}=\lambda U+h(\xi), \\
\mu_{ \pm}=\frac{1}{2}\left(-\epsilon \xi \pm \sqrt{\epsilon^{2} \xi^{2}+4 \lambda}\right) .
\end{array}
$$

For simplicity, let us think that $\lambda$ is real and positive. Then $\mu_{-}<0<\mu_{+}$. The system has exponential dichotomy with the gap $\mu_{+}-\mu_{-}=\sqrt{\epsilon^{2} \xi^{2}+4 \lambda} \approx \epsilon|\xi| \rightarrow \infty$ as $\xi \rightarrow \pm \infty$. However, as $\xi \rightarrow \infty, \mu_{+} \rightarrow 0_{+}$and as $\xi \rightarrow-\infty, \mu_{-} \rightarrow 0_{-}$. When the eigenvalues get closer to zero, estimate of the solution $h \rightarrow U$ by exponential dichotomies gets worse. The weight function $w(\xi)$ shifts the eigenvalues so that $\mu_{-}+\mu_{+}=0$ without reducing the gap between positive and negative exponents. See $\S 3$ for details.

Note that for functions in $E$,

$$
|U(\xi)|=O\left(e^{-(\sqrt{\epsilon} \xi)^{2} / 4}\right)=O\left(|U(\xi)| e^{-(x / \sqrt{\epsilon})^{2} / 4}\right) .
$$

The time for the function $U$ to decay to $e^{-1}$ is $\frac{2}{\sqrt{\epsilon}}$ in $\xi$ and is $2 \sqrt{\epsilon}$ in $x$. We hope to find better weight functions, like $w(\xi)=e^{-\epsilon \alpha \xi}$, so that the time scale of decay will be $O(1 / \epsilon)$ in $\xi$ and $O(1)$ in $x$. So far this has not been achieved.

Recall that the real $x$-axis is divided into three regions

$$
\mathbb{R}=\{x \leq-\bar{x}\} \cup\{-\bar{x} \leq x \leq \bar{x}\} \cup\{x \geq \bar{x}\} .
$$

In $\S 2$, we study system (1.5) in the middle region $|x|<\bar{x}$. Due the the possible presence of internal layers, the system is not slow-varying. However, we can show that if $|\lambda|$ is sufficiently large, the reduced system

$$
U_{\xi \xi}=\lambda U+h,
$$

has exponential dichotomy with large exponents. Since the coefficient $D f\left(u_{\epsilon}\right)-x I$ and its $\xi$-derivative are bounded in this region, if the largeness of the exponents exceeds the bound of the coefficients, then (1.5) has exponential dichotomy too. Here the boundedness of $|x|$ by $\bar{x}$ is important and is not valid in the two side regions. 
In $\S 3$, we study system (1.5) in the two side regions $x \leq-\bar{x}$ and $x \geq \bar{x}$. There is no internal layer. The system written in $\xi$ variable is slow-varying. By freezing $\xi$ and computing eigenvalues of the coefficient matrix, we can show that the system has exponential dichotomies in the two side regions $[\mathbf{3}]$.

In $\S 4$, using the exponential dichotomies, solutions of the spectrum problem (1.5) can be expressed by integral equations in each of the three regions, with some auxiliary data at their junction points. By the transverse intersection of stable and unstable subspaces at the junction points, the auxiliary data can be uniquely determined which yields a matched solution that is smooth in $\mathbb{R}$. The approach is similar to the shadowing lemma used in classical dynamical systems theory $[\mathbf{1 9}, \mathbf{1 4}, \mathbf{2 2}]$.

We note that if Hilbert space with weighted $L^{2}$ type norms are used, it is possible to show that $\mathcal{A}$ is $m$-sectorial in the sense of Kato [11]. Then $\mathcal{A}$ is sectorial as in our definition. Hilbert space method and $L^{2}$ type of norms are interesting alternatives to our approach but will not be discussed in this paper.

As a general rule, we always use the main branch of the square root. The following lemma will be used in both the middle and side regions.

Lemma 1.2. Let $s \geq 0$ be any real constant. If $\lambda \in \Sigma_{\pi / 4}$, then

(i) $\mid \sqrt{s^{2}+\lambda \mid} \geq \sqrt{|\lambda|} / \sqrt{2}$.

(ii) Consider the main branch of the square root. Then

$$
\operatorname{Re} \sqrt{s^{2}+\lambda} \geq \frac{\sqrt{|\lambda|}}{3} .
$$

Proof. (i) Let $\lambda=\sigma+i \omega$. First assume that $|\sigma| \leq|\omega|$. Then

$$
\mid \sqrt{s^{2}+\lambda \mid} \geq \sqrt{|\omega|} \geq \sqrt{|\lambda|} / \sqrt{2} .
$$

Next assume that $\sigma>|\omega|$, then

$$
\mid \sqrt{s^{2}+\lambda \mid} \geq \sqrt{|\lambda|} \geq \sqrt{|\lambda|} / \sqrt{2} .
$$

(ii) Let $z=\sqrt{s^{2}+\lambda}=x+i y$. Separating the real and imaginary part of $z^{2}=s^{2}+\lambda$, we have

$$
\begin{aligned}
& x^{2}-y^{2}=s^{2}+\sigma, \\
& 2 x y=\omega .
\end{aligned}
$$

Canceling $y$, we have

$$
\begin{array}{r}
x^{4}-\left(s^{2}+\sigma\right) x^{2}-(\omega / 2)^{2}=0, \\
x^{2}=\frac{1}{2}\left[s^{2}+\sigma+\sqrt{\left(s^{2}+\sigma\right)^{2}+\omega^{2}}\right] .
\end{array}
$$

First consider the case $\sigma \geq 0$. Then

$$
\begin{aligned}
x^{2} & \geq \frac{1}{2} \sqrt{\left(s^{2}+\sigma\right)^{2}+\omega^{2}} \\
& \geq|\lambda| / 2 . \\
x & \geq \sqrt{|\lambda|} / \sqrt{2} .
\end{aligned}
$$

Next consider the case $-|\omega| \leq \sigma<0$. For any $\omega \neq 0$, the function

$$
t \rightarrow t+\sqrt{t^{2}+\omega^{2}}, \quad t \in \mathbb{R},
$$


is strictly monotone. Thus, for a fixed $\omega \neq 0$, the minimum of $x^{2}$ is reached at the boundary of $\Sigma_{\pi / 4}$ where $\sigma=-|\omega|$ and $s^{2}+\sigma=s^{2}-|\omega| \geq-|\omega|$. Setting $t=s^{2}+\sigma$ and taking the lower limit, because $|\omega| \geq|\lambda| / \sqrt{2}$, we have

$$
\begin{aligned}
x^{2} & \geq \frac{1}{2}\left(-|\omega|+\sqrt{2 \omega^{2}}\right) \\
& =\frac{\sqrt{2}-1}{2}|\omega| \geq \frac{|\lambda|}{9}, \\
x & \geq \frac{\sqrt{|\lambda|}}{3} .
\end{aligned}
$$

\section{The Middle Region}

For convenience of notation, let $g=h$ in the middle region $|x|<\bar{x}$ so that (1.5) can be written as

$$
\begin{gathered}
W_{\xi}=A^{\text {mid }} W+B^{\text {mid }} W+\left(\begin{array}{l}
0 \\
g
\end{array}\right), \\
A^{\text {mid }}=\left(\begin{array}{cc}
0 & I \\
\lambda I & 0
\end{array}\right), \quad W=\left(\begin{array}{l}
U \\
V
\end{array}\right), \\
B^{m i d}=\left(\begin{array}{cc}
0 & 0 \\
D^{2} f\left(u_{\epsilon}\right) u_{\epsilon \xi} & D f\left(u_{\epsilon}\right)-\epsilon \xi I
\end{array}\right) .
\end{gathered}
$$

The system is a perturbation of $(2.2)$

$$
W_{\xi}=A^{m i d} W+\left(\begin{array}{l}
0 \\
g
\end{array}\right)
$$

The eigenvalues for (2.2) are $\mu= \pm \sqrt{\lambda}$. Assume that $\lambda \in \Sigma_{\pi / 4}-\{0\}$. By Lemma $1.2, \operatorname{Re} \sqrt{\lambda} \geq \sqrt{|\lambda|} / 3$. System (2.2) has an exponential dichotomy with $n$-dimensional stable and unstable subspaces spanned by $\left(\begin{array}{c}U \\ -\sqrt{\lambda} U\end{array}\right)$ and $\left(\begin{array}{c}U \\ \sqrt{\lambda} U\end{array}\right)$ respectively.

The eigenvectors of $A^{\text {mid }}$ form a $2 n \times 2 n$ matrix

$$
H^{\text {mid }}(\lambda):=\left(\begin{array}{cc}
I_{n} & I_{n} \\
-\sqrt{\lambda} I_{n} & \sqrt{\lambda} I_{n}
\end{array}\right) .
$$

For brevity, we drop the super-script mid on $H$. The first $n$ columns of $H(\lambda)$ are eigenvectors associated to the stable, and the last $n$ columns are eigenvectors associated to the unstable eigenvalues.

$$
H^{-1}(\lambda)=\left(\begin{array}{cc}
\frac{1}{2} I & -\frac{1}{2 \sqrt{\lambda}} I \\
\frac{1}{2} I & \frac{1}{2 \sqrt{\lambda}} I
\end{array}\right)
$$

Let $\tilde{P}=\left(\begin{array}{cc}0 & 0 \\ 0 & I_{n}\end{array}\right)$. The projection to the space spanned by the unstable eigenvectors is

$$
P_{u}(\lambda, x)=H \tilde{P} H^{-1}=\left(\begin{array}{cc}
\frac{1}{2} I & \frac{1}{2 \sqrt{\lambda}} I \\
\frac{\sqrt{\lambda}}{2} I & \frac{1}{2} I
\end{array}\right) .
$$


Note that $P_{u}$ is not uniformly bounded with respect to $\lambda$. This can be a problem in the estimate of the solution using exponential dichotomies and integral equations. In a similar work $[\mathbf{2 1}]$, the unboundedness of $P_{u}$ was circumvented by showing that

$$
\left|P_{s} \tilde{P}\right|+\left|P_{u} \tilde{P}\right| \leq C,
$$

uniformly with respect to $\lambda$.

In this paper, realizing that $W=\left(U, U_{\xi}\right)^{\top}$ may not be the best variable to rewrite a second order equation into a first order system, we will use coordinates on the stable and unstable subspaces as our phase variable.

Let $\mathcal{D}^{\text {mid }}(\lambda):=\operatorname{diag}\left(-\sqrt{\lambda} I_{n}, \sqrt{\lambda} I_{n}\right)$, and $W=(U, V)^{\top}$. Then $A^{\text {mid }}=H \mathcal{D}^{\text {mid }} H^{-1}$. Consider the $\lambda$-dependent change of variable $W=H Y$. System (2.1) becomes

$$
Y_{\xi}=\mathcal{D}^{\text {mid }} Y+H^{-1} B^{\text {mid }} H Y+H^{-1}\left(\begin{array}{l}
0 \\
g
\end{array}\right) \text {. }
$$

The differential equation (2.3) is a perturbation of the diagonalized system

$$
Y_{\xi}=\mathcal{D}^{\text {mid }} Y \text {. }
$$

That is, $Y_{j}^{\prime}=-\sqrt{\lambda} Y_{j}$, for $1 \leq j \leq n$, and $Y_{j}^{\prime}=\sqrt{\lambda} Y_{j}$, for $n+1 \leq j \leq 2 n$. It is easily verified that

$$
\begin{aligned}
& B^{\text {mid }}=\tilde{P} B^{\text {mid }}, \quad\left(\begin{array}{l}
0 \\
g
\end{array}\right)=\tilde{P}\left(\begin{array}{l}
0 \\
g
\end{array}\right), \\
& |H| \leq C \sqrt{|\lambda|}, \quad\left|H^{-1} \tilde{P}\right| \leq 1 / \sqrt{|\lambda|} .
\end{aligned}
$$

Therefore,

$$
\left|H^{-1} B^{m i d} H\right| \leq C, \quad\left|H^{-1}\left(\begin{array}{l}
0 \\
g
\end{array}\right)\right|=\frac{C|g|}{\sqrt{|\lambda|}} .
$$

For brevity, write (2.3) as

$$
\begin{aligned}
& Y_{\xi}=\mathcal{D}^{m i d} Y+\Theta Y+G, \\
& \text { where } \Theta:=H^{-1} B^{m i d} H, \quad G=H^{-1}\left(\begin{array}{l}
0 \\
g
\end{array}\right) \text {. }
\end{aligned}
$$

There exists a constant $C$, independent of $x$ and $\lambda$ such that

$$
|\Theta| \leq C, \quad|G|=\frac{C|g|}{\sqrt{|\lambda|}} .
$$

Let $Y=\left(Y_{1}, Y_{2}\right)^{\top}, \Theta=\left(\Theta_{1}, \Theta_{2}\right)^{\top}$ and $G=\left(G_{1}, G_{2}\right)^{\top}$. Then

$$
\begin{aligned}
Y_{1, \xi} & =-\sqrt{\lambda} Y_{1}+\Theta_{1} Y+G_{1}, \\
Y_{2, \xi} & =\sqrt{\lambda} Y_{2}+\Theta_{2} Y+G_{2} .
\end{aligned}
$$

Let $a=-\bar{x} / \epsilon, b=\bar{x} / \epsilon$. Solutions of (2.6) satisfy a system of integral equations:

$$
\begin{aligned}
& Y_{1}(\xi)=\int_{a}^{\xi} e^{-\sqrt{\lambda}(\xi-\zeta)}\left(\Theta_{1} Y+G_{1}\right) d \zeta+e^{-\sqrt{\lambda}(\xi-a)} Y_{1}(a), \\
& Y_{2}(\xi)=\int_{b}^{\xi} e^{\sqrt{\lambda}(\xi-\zeta)}\left(\Theta_{2} Y+G_{2}\right) d \zeta+e^{\sqrt{\lambda}(\xi-b)} Y_{2}(b) .
\end{aligned}
$$

¿From Lemma 1.2, $\operatorname{Re} \sqrt{\lambda} \geq \sqrt{|\lambda|} / 3$. We have

$$
\left|e^{-\sqrt{\lambda} \xi}\right|=e^{-\sqrt{|\lambda|} \xi / 3}, \text { for } \xi>0 .
$$


Assume that $\left(Y_{1}(a), Y_{2}(b)\right)$ is given. If $\sqrt{|\lambda|}>6\left(\left|\Theta_{1}\right|+\left|\Theta_{2}\right|\right)$, using the contraction mapping principle, $(2.7)$ has a unique solution

$$
\left(G_{1}, G_{2}, Y_{1}(a), Y_{2}(b)\right) \rightarrow\left(Y_{1}, Y_{2}\right)
$$

that satisfies

$$
\begin{aligned}
& \left|Y_{1}\right| \leq \frac{3}{\sqrt{|\lambda|}}\left(\left|\Theta_{1} Y\right|+G_{1} \mid\right)+\left|Y_{1}(a)\right|, \\
& \left|Y_{2}\right| \leq \frac{3}{\sqrt{|\lambda|}}\left(\left|\Theta_{2} Y\right|+G_{2} \mid\right)+\left|Y_{2}(b)\right| .
\end{aligned}
$$

Combining them, we have

$$
|Y| \leq \frac{C}{\sqrt{|\lambda|}}\left(\left|G_{1}\right|+\left|G_{2}\right|\right)+\left|Y_{1}(a)\right|+\left|Y_{2}(b)\right| .
$$

From the estimates for $\left|G_{1}\right|+\left|G_{2}\right|,(2.5)$, we have

$$
|Y| \leq \frac{C|g|}{|\lambda|}+\left|Y_{1}(a)\right|+\left|Y_{2}(b)\right|
$$

\section{The Side Regions}

Consider the two side regions $x<-\bar{x}$ and $x>\bar{x}$. Let $\alpha=\epsilon / 4$. We make the following change of variables to eliminate the term $\epsilon \xi U_{\xi}$ in (1.5).

$$
\begin{aligned}
U & =e^{-\alpha \xi^{2}} \bar{U}, \quad h=e^{-\alpha \xi^{2}} g, \\
U_{\xi} & =e^{-\alpha \xi^{2}}\left(\bar{U}_{\xi}-2 \alpha \xi \bar{U}\right), \\
U_{\xi \xi} & =e^{-\alpha \xi^{2}}\left(\bar{U}_{\xi \xi}-4 \alpha \xi \bar{U}_{\xi}+\left(4 \alpha^{2} \xi^{2}-2 \alpha\right) \bar{U}\right), \\
U_{\xi \xi}+\epsilon \xi U_{\xi} & =e^{-\alpha \xi^{2}}\left(\bar{U}_{\xi \xi}+(\epsilon \xi-4 \alpha \xi) \bar{U}_{\xi}+\left(4 \alpha^{2} \xi^{2}-2 \alpha-2 \alpha \epsilon \xi^{2}\right) \bar{U}\right) \\
& =e^{-\alpha \xi^{2}}\left(\bar{U}_{\xi \xi}-\left(\epsilon^{2} \xi^{2} / 4+\epsilon / 2\right) \bar{U}\right) .
\end{aligned}
$$

System (1.5) becomes

Write (3.1) as a first order system

$$
\begin{gathered}
W_{\xi}=A^{s d} W+B^{s d} W+\left(\begin{array}{l}
0 \\
g
\end{array}\right), \quad W=(\bar{U}, \bar{V})^{\top}, \\
A^{s d}=\left(\begin{array}{cc}
0 & I \\
\left(\lambda+x^{2} / 4\right) I-(x / 2) D f\left(u_{\epsilon}\right) & 0
\end{array}\right), \\
B^{s d}=\left(\begin{array}{cc}
0 & 0 \\
D^{2} f\left(u_{\epsilon}\right) u_{\epsilon \xi}+(\epsilon / 2) I & D f\left(u_{\epsilon}\right)
\end{array}\right) .
\end{gathered}
$$

If the conservation law is strictly hyperbolic, then for $|x|>\bar{x}$, the eigenvalues of $D f\left(u_{\epsilon}\right)$ are real and distinct. In the sequel, we assume that $D f\left(u_{\epsilon}\right)$ has simple eigenvalues. This can be achieved by adding a small perturbation to $D f\left(u_{\epsilon}\right)$, without interfering with the proof of $\mathcal{A}$ being sectorial. Denote the eigenvalues of $D f\left(u_{\epsilon}\right)$ by $\nu_{1}, \ldots, \nu_{n}$, and the corresponding eigenvectors by $\mathbf{r}_{1}, \ldots, \mathbf{r}_{n}$.

The homogeneous part of (3.2) can be viewed as a perturbation of the system

$$
W_{\xi}=A^{s d} W
$$


System (3.3) is slow-varying. Let $\mu$ be an eigenvalue of $A^{\text {sd }}$. It is easily verified that

$$
\operatorname{det}\left[\mu^{2}-\left(\lambda+x^{2} / 4\right) I+(x / 2) D f\left(u_{\epsilon}\right)\right]=0 .
$$

Therefore one of the following equations must hold:

$$
\mu^{2}=\lambda+x^{2} / 4-(x / 2) \nu_{j}, \quad j=1, \ldots, n .
$$

Thus, there are two eigenvalues of $A^{s d}$ for each $j$ :

$$
\begin{aligned}
\mu_{j}^{ \pm} & = \pm \sqrt{x^{2} / 4-(x / 2) \nu_{j}+\lambda} \\
& = \pm \sqrt{\left(x-\nu_{j}\right)^{2} / 4-\nu_{j}^{2} / 4+\lambda},
\end{aligned}
$$

with corresponding eigenvectors

$$
\left(\mathbf{r}_{j}, \mu_{j}^{ \pm} \mathbf{r}_{j}\right)^{\top}
$$

Without the loss of generality, assume that $|\bar{x}|>2\left|\nu_{j}\right|$. Then $\left(x-\nu_{j}\right)^{2} / 4-$ $\nu_{j}^{2} / 4=x\left(x-2 \nu_{j}\right) / 4>0$ for $|x| \geq \bar{x}$. Let

$$
r_{j}:=\sqrt{\left(x-\nu_{j}\right)^{2} / 4-\nu_{j}^{2} / 4}>0 .
$$

If $\lambda \in \Sigma_{\pi / 4}$, then from Lemma 1.2,

$$
\mu_{j}^{ \pm}= \pm \sqrt{r_{j}^{2}+\lambda}, \quad\left|\operatorname{Re} \mu_{j}^{ \pm}\right| \geq \sqrt{|\lambda|} / 3 .
$$

Let $\mathbf{R}=\left(\mathbf{r}_{1} \ldots \mathbf{r}_{n}\right)$ and $\mathcal{M}(\lambda, x)=\operatorname{diag}\left(\mu_{1}^{+} \ldots \mu_{n}^{+}\right)$be $n \times n$ matrices. The eigenvectors of $A$ form a $2 n \times 2 n$ matrix

$$
H^{s d}(\lambda, x):=\left(\begin{array}{cc}
\mathbf{R} & 0 \\
0 & \mathbf{R}
\end{array}\right)\left(\begin{array}{cc}
I_{n} & I_{n} \\
-\mathcal{M} & \mathcal{M}
\end{array}\right) .
$$

For brevity, we will drop the super-script $s d$ in this section. The first $n$ columns of $H$ are eigenvectors $\left(\mathbf{r}_{j}, \mu_{j}^{-} \mathbf{r}_{j}\right)^{\top}$ for the corresponding $\mu_{j}^{-}$, and the last $n$ columns are eigenvectors $\left(\mathbf{r}_{j}, \mu_{j}^{+} \mathbf{r}_{j}\right)^{\top}$ for the corresponding $\mu_{j}^{+}$.

$$
H^{-1}=\left(\begin{array}{cc}
\frac{1}{2} I & -\frac{1}{2} \mathcal{M}^{-1} \\
\frac{1}{2} I & \frac{1}{2} \mathcal{M}^{-1}
\end{array}\right)\left(\begin{array}{cc}
\mathbf{R}^{-1} & 0 \\
0 & \mathbf{R}^{-1}
\end{array}\right) .
$$

Let $\tilde{P}=\left(\begin{array}{cc}0 & 0 \\ 0 & I_{n}\end{array}\right)$. The projection to the space spanned by the unstable eigenvectors is

$$
P_{u}(\lambda, x)=H \tilde{P} H^{-1}=\left(\begin{array}{cc}
\mathbf{R} & 0 \\
0 & \mathbf{R}
\end{array}\right)\left(\begin{array}{cc}
\frac{1}{2} I & \frac{1}{2} \mathcal{M}^{-1} \\
\frac{1}{2} \mathcal{M} & \frac{1}{2} I
\end{array}\right)\left(\begin{array}{cc}
\mathbf{R}^{-1} & 0 \\
0 & \mathbf{R}^{-1}
\end{array}\right) .
$$

Note that as $|x|$ and $\lambda$ can go to infinity, $\left|P_{u}(\lambda, x)\right|$ is not uniformly bounded with respect to $|x|$ and $\lambda$ due to the entry $\frac{1}{2} \mathcal{M}$. Geometrically, this is due to the small angle between the stable and unstable subspace if $\mathcal{M}$ is large. Similar to $\S 2$, we will make the change of variables so that the coordinates $\left(Z_{1}, Z_{2}\right)$ on stable and unstable subspaces will be the phase variable.

Let $\mathcal{D}^{s d}(\lambda, x):=\operatorname{diag}(-\mathcal{M}, \mathcal{M})$ and $W=(U, V)^{\top}$. Then $A^{s d}=H \mathcal{D}^{s d} H^{-1}$. Consider the $(\lambda, x)$-dependent change of variables $W=H Z$. System (3.2) becomes

$$
Z_{\xi}=\mathcal{D}^{s d} Z+\left(H^{-1} B^{s d} H-H^{-1} H_{\xi}\right) Z+H^{-1}\left(\begin{array}{l}
0 \\
g
\end{array}\right) \text {. }
$$


The differential equation (3.4) is a perturbation of the diagonalized system

$$
Z_{\xi}=\mathcal{D}^{s d} Z
$$

That is, $Z_{j}^{\prime}=\mu_{j}^{-} Z_{j}$, if $1 \leq j \leq n$, and $Z_{j}^{\prime}=\mu_{j-n}^{+} Z_{j}$, if $n+1 \leq j \leq 2 n$. System (3.5) has an exponential dichotomy with projection $\tilde{P}$ and a large spectral gap if $x>\bar{x}, \lambda \in \Sigma_{\pi / 4}$ and $|\lambda|$ is sufficiently large. It is easily verified that

$$
\begin{aligned}
& B=\tilde{P} B, \quad\left(\begin{array}{l}
0 \\
g
\end{array}\right)=\tilde{P}\left(\begin{array}{l}
0 \\
g
\end{array}\right), \\
& \left|H^{-1} \tilde{P}\right| \leq C\left|\mathcal{M}^{-1}\right|, \quad|H| \leq C|\mathcal{M}|, \\
& |\mathcal{M}| \leq C \sqrt{\max _{j}\left\{\left|r_{j}^{2}+\lambda\right|\right\}}, \\
& \left|\mathcal{M}^{-1}\right| \leq C / \sqrt{\min _{j}\left\{\left|r_{j}^{2}+\lambda\right|\right\}} .
\end{aligned}
$$

Therefore,

$$
\left|H^{-1} B^{s d} H\right| \leq C, \quad\left|H^{-1}\left(\begin{array}{l}
0 \\
g
\end{array}\right)\right|=\frac{C}{\sqrt{\min _{j}\left\{\left|r_{j}^{2}+\lambda\right|\right\}}}|g| .
$$

It is easily verified that there is a constant $C$ such that $\left|H^{-1}\right| \leq C$ uniformly with respect to $(\lambda, x)$. Moreover, using $x=\epsilon \xi$, we have

$$
\partial \mu_{j} / \partial \xi=\frac{\epsilon\left(x-\nu_{j}\right)}{4 \sqrt{\left(x-\nu_{j}\right)^{2} / 4-\nu_{j}^{2} / 4+\lambda}}=O(\epsilon)
$$

for all $(j, \lambda, x)$. Therefore, $H^{-1} H_{\xi}=O(\epsilon)$.

System (3.4) can be written as

$$
\begin{aligned}
Z_{\xi} & =\mathcal{D}^{s d} Z+\mathcal{N} Z+G, \\
\text { where } \mathcal{N}: & =H^{-1} B^{s d} H-H^{-1} H_{\xi}, \quad G=H^{-1}\left(\begin{array}{l}
0 \\
g
\end{array}\right) .
\end{aligned}
$$

We have shown that there exists a constant $C$, independent of $x$ and $\lambda$ such that

$$
|\mathcal{N}| \leq C, \quad|G|=\frac{C}{\sqrt{|\lambda|}}|g| .
$$

Let $Z=\left(Z_{1}, Z_{2}\right)^{\top}$ and $G=\left(G_{1}, G_{2}\right)^{\top}$. Then

$$
\begin{aligned}
Z_{1, \xi} & =-\mathcal{M} Z_{1}+\mathcal{N}_{1} Z+G_{1}, \\
Z_{2, \xi} & =\mathcal{M} Z_{2}+\mathcal{N}_{2} Z+G_{2} .
\end{aligned}
$$

Based on this and the exponential dichotomies for (3.5) for $|x|>\bar{x}$, we will show that if $\left(Z_{2}(a), Z_{1}(b)\right)$ is given, there is a unique solution $Z$ to (3.7) in the two side regions.

For definitiveness, we consider the interval $x \geq \bar{x}$, since the interval $x \leq-\bar{x}$ can be handled similarly. 
In $(b, \infty)$, solutions of $(3.7)$ can be expressed as a system of integral equations:

$$
\begin{aligned}
& Z_{1}(\xi)=\int_{b}^{\xi} e^{-\int_{\zeta}^{\xi} \mathcal{M}(\tau) d \tau}\left(\mathcal{N}_{1} Z+G_{1}\right) d \zeta+e^{-\int_{b}^{\xi} \mathcal{M}(\tau) d \tau} Z_{1}(b), \\
& Z_{2}(\xi)=\int_{\infty}^{\xi} e^{\int_{\zeta}^{\xi} \mathcal{M}(\tau) d \tau}\left(\mathcal{N}_{2} Z+G_{2}\right) d \zeta
\end{aligned}
$$

Using $\left|\operatorname{Re} \mu_{j}^{ \pm}\right|>\sqrt{|\lambda|} / 3$, we have

$$
\left|e^{-\int_{\zeta}^{\xi} \mathcal{M}(\tau) d \tau}\right| \leq e^{-\sqrt{|\lambda|}|\xi-\zeta| / 3}, \xi>\zeta .
$$

Assume that $Z_{1}(b)$ is given. If $\sqrt{|\lambda|}>6\left(\left|\mathcal{N}_{1}\right|+\left|\mathcal{N}_{2}\right|\right)$, the fixed point problem (3.8) has a unique solution

$$
\left(G_{1}, G_{2}, Z_{1}(b)\right) \rightarrow\left(Z_{1}, Z_{2}\right)
$$

that satisfies

$$
\begin{aligned}
& \left|Z_{1}\right| \leq \frac{3}{\sqrt{|\lambda|}}\left(\left|\mathcal{N}_{1} Z+G_{1}\right|\right)+\left|Z_{1}(b)\right|, \\
& \left|Z_{2}\right| \leq \frac{3}{\sqrt{|\lambda|}}\left(\left|\mathcal{N}_{2} Z+G_{2}\right|\right)
\end{aligned}
$$

Combining them, we have

$$
|Z| \leq \frac{C}{\sqrt{|\lambda|}}\left(\left|G_{1}\right|+\left|G_{2}\right|\right)+\left|Z_{1}(b)\right|
$$

From the estimates for $\left|G_{1}\right|+\left|G_{2}\right|$, (3.6), we have

$$
|Z| \leq \frac{C|g|}{|\lambda|}+\left|Z_{1}(b)\right| \text {. }
$$

Similarly, in the region $(-\infty, a)$, assume that $Z_{2}(a)$ is given, then if $\sqrt{|\lambda|}>$ $6\left(\left|\mathcal{N}_{1}\right|+\left|\mathcal{N}_{2}\right|\right)$, there exists a unique solution

$$
\left(G_{1}, G_{2}, Z_{2}(a)\right) \rightarrow\left(Z_{1}, Z_{2}\right)
$$

to (3.7) such that

$$
|Z| \leq \frac{C|g|}{|\lambda|}+\left|Z_{2}(a)\right|
$$

\section{Matching of solutions in the middle and side regions}

The purpose of this section is to combine solutions in the middle and side regions and to complete the proof of Theorem 1.1.

Recall that the three regions are separated by

$$
-\infty<a<b<\infty, \quad a=-\bar{x} / \epsilon, b=\bar{x} / \epsilon .
$$

In order to have a smooth solution defined in $\mathbb{R}$, we need to match $W=(U, V)^{\top}$ in the side and middle regions at the junction points $\xi=a$ and $b$. Using $a \pm$ and $b \pm$ to distinguish the side and middle regions, we have

$$
\left.W\right|_{a+}=\left.W\right|_{a-},\left.\quad W\right|_{b+}=\left.W\right|_{b-} .
$$


Recall that

$$
\begin{aligned}
W^{\text {mid }} & =\left(\begin{array}{cc}
I & I \\
-\sqrt{\lambda} I & \sqrt{\lambda} I
\end{array}\right)\left(\begin{array}{l}
Y_{1} \\
Y_{2}
\end{array}\right)=\left(\begin{array}{c}
Y_{1}+Y_{2} \\
\sqrt{\lambda}\left(Y_{2}-Y_{1}\right)
\end{array}\right), \\
W^{s d} & =\left(\begin{array}{cc}
R & 0 \\
0 & R
\end{array}\right)\left(\begin{array}{cc}
I & I \\
-\mathcal{M} & \mathcal{M}
\end{array}\right)\left(\begin{array}{l}
Z_{1} \\
Z_{2}
\end{array}\right)=\left(\begin{array}{c}
R\left(Z_{1}+Z_{2}\right) \\
R \mathcal{M}\left(Z_{2}-Z_{1}\right)
\end{array}\right) .
\end{aligned}
$$

We must have

$$
\begin{aligned}
R\left(Z_{1}+Z_{2}\right)(a-) & =\left(Y_{1}+Y_{2}\right)(a+), & R \mathcal{M}\left(Z_{2}-Z_{1}\right)(a-) & =\sqrt{\lambda}\left(Y_{2}-Y_{1}\right)(a+), \\
R\left(Z_{1}+Z_{2}\right)(b+) & =\left(Y_{1}+Y_{2}\right)(b-), & R \mathcal{M}\left(Z_{2}-Z_{1}\right)(b+) & =\sqrt{\lambda}\left(Y_{2}-Y_{1}\right)(b-) .
\end{aligned}
$$

Setting $\mathcal{M} / \sqrt{\lambda}=\overline{\mathcal{M}}, R^{-1} Y_{j}=\bar{Y}_{j}, j=1,2$, we have

$$
\begin{aligned}
Z_{2}(a-)-\bar{Y}_{1}(a+) & =-Z_{1}(a-)+\bar{Y}_{2}(a+), \\
\overline{\mathcal{M}} Z_{2}(a-)+\bar{Y}_{1}(a+) & =\overline{\mathcal{M}} Z_{1}(a-)+\bar{Y}_{2}(a+), \\
Z_{1}(b+)-\bar{Y}_{2}(b-) & =-Z_{2}(b+)+\bar{Y}_{1}(b-), \\
\overline{\mathcal{M}} Z_{1}(b+)+\bar{Y}_{2}(b-) & =\overline{\mathcal{M}} Z_{2}(b+)+\bar{Y}_{1}(b-) .
\end{aligned}
$$

Solving for $\left(Z_{2}(a-), \bar{Y}_{1}(a+), Z_{1}(b+), \bar{Y}_{2}(b-)\right)$, we have

$$
\begin{aligned}
Z_{2}(a-) & =(\overline{\mathcal{M}}+I)^{-1}\left[(\overline{\mathcal{M}}-I) Z_{1}(a-)+2 \bar{Y}_{2}(a+)\right], \\
\bar{Y}_{1}(a+) & =(\overline{\mathcal{M}}+I)^{-1}\left[2 \overline{\mathcal{M}} Z_{1}(a-)+(I-\overline{\mathcal{M}}) \bar{Y}_{2}(a+)\right], \\
Z_{1}(b+) & =(\overline{\mathcal{M}}+I)^{-1}\left[(\overline{\mathcal{M}}-I) Z_{2}(b+)+2 \bar{Y}_{1}(b-)\right], \\
\bar{Y}_{2}(b-) & =(\overline{\mathcal{M}}+I)^{-1}\left[2 \overline{\mathcal{M}} Z_{2}(b+)+(I-\overline{\mathcal{M}}) \bar{Y}_{1}(b-)\right] .
\end{aligned}
$$

Based on (2.8), (3.9) and (3.10), the right hand sides of (4.1) also depend on

$$
\left(Z_{2}(a-), \bar{Y}_{1}(a+), \bar{Y}_{2}(b-), Z_{1}(b+)\right) .
$$

Written as an abstract equation,

$$
\left(Z_{2}(a-), \bar{Y}_{1}(a+), \bar{Y}_{2}(b-), Z_{1}(b+)\right)=\mathcal{F}\left(\left(Z_{2}(a-), \bar{Y}_{1}(a+), \bar{Y}_{2}(b-), Z_{1}(b+), g\right),\right.
$$

system (4.1) can be viewed as a fixed point problem. We show that the right hand side is a contraction mapping with respect to $\left(Z_{2}(a-), \bar{Y}_{1}(a+), \bar{Y}_{2}(b-), Z_{1}(b+)\right)$.

Using Lemma 1.2, (i), we have

$$
\begin{aligned}
\left|(\overline{\mathcal{M}}+I)^{-1}\right| & \leq C, \\
\left|(\overline{\mathcal{M}}+I)^{-1} \overline{\mathcal{M}}\right| & =\left|I-(\overline{\mathcal{M}}+I)^{-1}\right| \leq C .
\end{aligned}
$$

From this, we have

$$
\mid \mathcal{F}\left(\left(Z_{2}(a-), \bar{Y}_{1}(a+), \bar{Y}_{2}(b-), Z_{1}(b+), g\right) \mid \leq C\left(\left|Z_{1}(a-)\right|+\left|\bar{Y}_{2}(a+)\right|+\left|\bar{Y}_{1}(b-)\right|+\left|Z_{2}(b+)\right|\right)\right. \text {. }
$$

For any $\delta>0$, there exists $\lambda_{M}>0$ such that if $|\lambda| \geq \lambda_{M}$, then from (3.8), (3.9) and (3.6), we have

$$
\left|Z_{2}(b+)\right| \leq \delta\left|Z_{1}(b+)\right|+\frac{C|g|}{|\lambda|} .
$$

Similarly if $\lambda_{M}$ is sufficiently large, for $|\lambda| \geq \lambda_{M}$,

$$
\left|Z_{1}(a-)\right| \leq \delta\left|Z_{2}(a-)\right|+\frac{C|g|}{|\lambda|} .
$$


For any $\delta>0$, if $\epsilon$ is sufficiently small so that $b-a$ is sufficiently large, and if $|\lambda|$ is sufficiently large, from (2.7), (2.8) and (2.5), we have

$$
\left|\bar{Y}_{1}(b-)\right|+\left|\bar{Y}_{2}(a+)\right| \leq \delta\left(\left|\bar{Y}_{1}(a+)\right|+\left|\bar{Y}_{2}(b-)\right|\right)+\frac{C|g|}{|\lambda|} .
$$

Therefore,

$$
\begin{aligned}
& \mid \mathcal{F}\left(\left(Z_{2}(a-), \bar{Y}_{1}(a+), \bar{Y}_{2}(b-), Z_{1}(b+), g\right) \mid\right. \\
\leq & C \delta\left(\left|Z_{2}(a-)\right|+\left|\bar{Y}_{1}(a+)\right|+\left|\bar{Y}_{2}(b-)\right|+\left|Z_{1}(b+)\right|\right)+\frac{C|g|}{|\lambda|} .
\end{aligned}
$$

Thus if $\delta$ is sufficiently small, then $\mathcal{F}$ is a contraction and there exists a unique fixed point for (4.1). Moreover,

$$
\left|\left(Z_{2}(a-), \bar{Y}_{1}(a+), \bar{Y}_{2}(b-), Z_{1}(b+)\right)\right| \leq \frac{C|g|}{|\lambda|} .
$$

Substituting into (2.8), (3.9) and (3.10), we have

$$
\begin{aligned}
& |Y| \leq \frac{C|g|}{|\lambda|} \quad \text { in the middle region and } \\
& |Z| \leq \frac{C|g|}{|\lambda|} \quad \text { in the side regions. }
\end{aligned}
$$

Recall that $U$ is the first row of $W=(U, V)^{\top}$. In the middle region,

$$
U=\left(\begin{array}{ll}
I_{n \times n} & 0
\end{array}\right) H^{m i d} Y=\left(Y_{1}+Y_{2}\right) .
$$

Therefore, we have

$$
|U| \leq C|Y| \leq \frac{C|g|}{|\lambda|} \leq \frac{C|h|}{|\lambda|}
$$

In the two side regions, we have

$$
\bar{U}=\left(\begin{array}{ll}
I_{n \times n} & 0
\end{array}\right) H^{s d} Z=\mathbf{R}\left(Z_{1}+Z_{2}\right) .
$$

Therefore, we have

$$
|\bar{U}| \leq C|Z| \leq \frac{C|g|}{|\lambda|} .
$$

Recall that $U=e^{-\alpha \xi^{2}} \bar{U}$ and $h=e^{-\alpha \xi^{2}} g$ defined at the beginning of $\S 3$. We have in the side regions

$$
\sup _{|\xi| \geq \bar{x} / \epsilon}|U(\xi)| e^{\epsilon \xi^{2} / 4} \leq \frac{C|g|}{|\lambda|} \leq \frac{C}{|\lambda|} \sup _{|\xi| \geq \bar{x} / \epsilon}|h(\xi)| e^{\epsilon \xi^{2} / 4} .
$$

Combining estimates (4.2) and (4.3), we have shown that there exists $\lambda_{M}>0$ such that if $\lambda \in \Sigma_{\pi / 4}$ and if $|\lambda| \geq \lambda_{M}>0$, then $\lambda \in \rho(\mathcal{A})$. Moreover, $U \in E$ and

$$
\|U\|_{E} \leq C\|h\|_{E} /|\lambda| \text {. }
$$

The proof of the following lemma is elementary and will not be rendered here.

Lemma 4.1. Assume that for some $\lambda_{M}>0$, the set

$$
S=\left\{\lambda\left|\lambda \in \Sigma_{\pi / 4} \cap\right| \lambda \mid \geq \lambda_{M}\right\}
$$

is in $\rho(\mathcal{A})$, and

$$
\left\|(\lambda-\mathcal{A})^{-1}\right\| \leq M /|\lambda|, \quad \lambda \in S
$$


Then

$$
\Sigma_{\pi / 4}+\sqrt{2} \lambda_{M} \subset \rho(\mathcal{A})
$$

with

$$
\left\|(\lambda-\mathcal{A})^{-1}\right\| \leq \frac{\sqrt{2} M}{\left|\lambda-\sqrt{2} \lambda_{M}\right|}, \quad \lambda \in \Sigma_{\pi / 4}+\sqrt{2} \lambda_{M} .
$$

Based on Lemma 4.1, $\mathcal{A}$ is sectorial in the function space $E$.

The proof of Theorem 1.1 has been completed.

\section{References}

[1] H. Berens and P. L. Butzer, semigroup of operators and approximation, Springer, Berlin, 1967.

[2] S.-N. Chow, X.-B. Lin and K. Palmer, A shadowing lemma with applications to semilinear parabolic equations, SIAM J. Math. Anal. 20 (1989), 547-557.

[3] W. A. Coppel, Dichotomies in stability theory, Lecture Notes in Mathematics, 629, Springer, 1978.

[4] C. M. Dafermos, Solution of the Riemann problem for a class of hyperbolic systems of conservation laws by the viscosity method, Arch. Ration. Mech. Anal. 52 (1973), 1-9.

[5] G. Da Prato and P. Grisvard, Equations d'evolution abstraites non linearéaires de type parabolique, Ann. Mat. Pura Appl. 120 (1979), 329-396.

[6] A. Friedman, Partial differential equations, Holt. Reinhart and Winston, New York, 1969.

[7] R. Gardner and K. Zumbrun, The gap lemma and geometric criteria for instability of viscous shock profiles, Comm. Pure Appl. Math., 51 (1998), pp. 789-847.

[8] C. K. R. T. Jones, Geometric singular perturbation theory, Lecture Notes in Math. 1609, Springer-Verlag, Berlin, 1995, 44-118.

[9] D. Henry, Geometric theory of semilinear parabolic equations, Lecture Notes in Math. 840, Springer-Verlag, 1981.

[10] T. Kapitula and B. Sandstede, Stability of bright solitary-wave solutions to perturbed nonlinear Schrödinger equations, Phys. D, 124 (1998), pp. 58-103.

[11] T. Kato, Perturbation theory for linear operators, Springer-Verlag, New York, 1966.

[12] M. Lewicak, Stability conditions for patterns of noninteracting large shock waves, SIAM J. Math. Anal. 32 (2001), 1094-1116.

[13] M. Lewicka, A note on the spectral stability conditions for shock patterns, preprint.

[14] X.-B. Lin, Using Melnikov's method to solve Silnikov's problems," Proc. Royal Soc. Edinburgh, 116A (1990), 295-325.

[15] X.-B. Lin, Exponential dichotomies in intermediate spaces with applications to a diffusively perturbed predator-prey model, J. Differential Equations, 108 (1994), 36-63.

[16] T.-P. Liu, Pointwise convergence to shock waves for viscous conservation laws, Comm. Pure Appl. Math., 50 (1997), pp. 1113-1182.

[17] T.-P. Liu and K. Zumbrun, Nonlinear stability of an undercompressive shock for complex Burgers equation, Comm. Math. Phys., 168 (1995), pp. 163-186.

[18] T.-P. Liu and K. Zumbrun, On nonlinear stability of general undercompressive viscous shock waves, Comm. Math. Phys., 174 (1995), pp. 319-345.

[19] K. J. Palmer, Exponential dichotomies and transversal homoclinic points, J. Differential Equations, 55 (1984), 225-256.

[20] A. Pazy, semigroup of linear operators and applications to partial differential equations, Springer, New York, 1983.

[21] X.-B. Lin and S. Schecter, Stability of self-similar solutions of the Dafermos regularization of a system of conservation laws, SIAM J. Math. Anal. 35 (2003), 884-921.

[22] Björn Sandstede, Verzweigungstheorie homokliner Verdopplungen, thesis, University of Stuttgart, 1993.

[23] S. Schecter, D. Marchesin and B. J. Plohr, Structurally stable Riemann solutions, J. Differential Equations, 126 (1996), 303-354.

[24] S. Schecter, Undercompressive shock waves and the Dafermos regularization, Nonlinearity 15 (2002), 1361-1377. 
[25] S. Schochet, Sufficient conditions for local existence via Glimn's scheme for large B.V. data, J. Differential Equations, 89 (1991), 317-354.

[26] P. Szmolyan, personal communication.

[27] V. A. Tupčiev, On the splitting of an arbitrary discontinuity for a system of two first-order quasi-linear equations, USSR Comput. Math. Phys., 4(1964), 36-48.

[28] V. A. Tupčiev, The method of introducing a viscosity in the study of a problem of decay of a discontinuity, Soviet Math. Dokl., 14 (1973), 978-982.

[29] A. E. Tzavaras, Wave interactions and variation estimates for self-similar zero-viscosity limits in systems of conservation laws, Arch. Ration. Mech. Anal. 135 (1996), 1-60.

[30] K. Zumbrun and P. Howard, Pointwise semigroup methods and stability of viscous shock waves, Indiana Univ. Math. J., 47 (1998), pp. 741-871.

Department of Mathematics, North Carolina State University, Raleigh, NC 276958205

E-mail address: xblin@math.ncsu.edu 